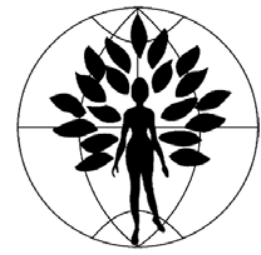

www.figo.org

\title{
Prolapse severity, symptoms and impact on quality of life among women planning sacrocolpopexy
}

\author{
M.P. FitzGerald ${ }^{a, *}$, N.K. Janz ${ }^{\text {b }}$, P.A. Wren ${ }^{\text {c }}$, J.T. Wei ${ }^{b}$, A.M. Weber ${ }^{d}$, \\ C. Ghetti ${ }^{e}$, G.W. Cundiff ${ }^{f}$ \\ for the Pelvic Floor Disorders Network ${ }^{g}$
}

a Loyola University Medical Center, Maywood, IL, USA

b University of Michigan, Ann Arbor, MI, USA

c Oakland University, Rochester, MI, USA

d Project Scientist for the PFDN, from the Contraception and Reproductive Health Branch, Center for Population Research, National Institute of Child Health and Human Development, Bethesda, MD, USA

e University of Pittsburgh Magee-Women's Hospital, Pittsburgh, PA, USA

f Johns Hopkins School of Medicine, Baltimore, MD, USA

${ }^{\mathrm{g}}$ National Institute of Child Health and Human Development, Bethesda, MD, USA

Received 30 November 2006; received in revised form 12 March 2007; accepted 15 March 2007

\section{KEYWORDS}

Pelvic organ prolapse;

Quality of life;

Pelvic floor dysfunction

\begin{abstract}
Objectives: To explore the relationship between severity of pelvic organ prolapse (POP), symptoms of pelvic dysfunction and quality of life using validated measures. Method: Baseline data from 314 participants in the Colpopexy And Urinary Reduction Efforts (CARE) trial were analyzed. Pelvic symptoms and impact were assessed using the Pelvic Floor Distress Inventory (PFDI) and the Pelvic Floor Impact Questionnaire (PFIQ). PFDI and PFIQ scores were compared by prolapse stage and history of incontinence or POP surgery. Regression analyses were performed to identify other predictors of symptoms and impact. Results: Women were predominantly (90\%) Caucasian and had mean age of 61 years. Women with stage II POP, especially those with prior surgery, reported more symptoms and impact than women with more advanced POP. There were no other significant predictors of symptoms or life impact. Conclusions: Women planning sacrocolpopexy with stage II prolapse and prior pelvic surgery reported more symptoms and quality of life impact than those with more advanced prolapse.

(c) 2007 International Federation of Gynecology and Obstetrics. Published by Elsevier Ireland Ltd. All rights reserved.
\end{abstract}

\footnotetext{
is Presented at Society of Gynecologic Surgeons 31st Annual Scientific Meeting, 2005.

* Corresponding author. Division of Female Pelvic Medicine and Reconstructive Surgery, Loyola University Medical Center, 2160 South First Avenue, Bldg 103, Room 1004, Maywood, IL 60153, USA.

E-mail address: Mfitzg8@lumc.edu (M.P. FitzGerald).
}

0020-7292/\$ - see front matter (c) 2007 International Federation of Gynecology and Obstetrics. Published by Elsevier Ireland Ltd. All rights reserved. 


\section{Introduction}

Pelvic organ prolapse (POP) is common, with approximately 200,000 inpatient surgical procedures performed in the United States annually for its treatment [1]. Many women with POP experience pelvic symptoms including urinary, colorectal and sexual dysfunction; however, others, including some with advanced POP, report few such pelvic symptoms.

Published studies of the relationship between prolapse severity and pelvic symptoms demonstrate a relatively weak relationship between those variables, but previous studies have been limited by their retrospective nature and by their use of poorly validated or unvalidated questionnaires. The Colpopexy And Urinary Reduction Efforts (CARE) study is an ongoing randomized clinical trial conducted by the Pelvic Floor Disorders Network (PFDN) in which women with POP but without symptoms of stress urinary incontinence undergoing abdominal sacrocolpopexy were randomly assigned to either concomitant Burch or no Burch [2,3]. Prior to surgery, participants completed several validated symptom and quality of life measures, including the Pelvic Floor Distress Inventory (PFDI) and the Pelvic Floor Impact Questionnaire (PFIQ) [4].

The aim of this analysis was to explore the relationship between severity of POP and symptoms of pelvic dysfunction and quality of life using validated measures among women in the CARE trial before surgery.

\section{Materials and methods}

The Pelvic Floor Disorders Network (PFDN) is a cooperative network of investigators from participating clinical centers and a Data Coordinating Center (DCC), supported by the National Institute of Child Health and Human Development (NICHD). The primary goal of the PFDN is to improve the level of knowledge about pelvic floor disorders in women, including pelvic organ prolapse, urinary incontinence and fecal incontinence. The CARE protocol was approved by the Institutional Review Boards at all clinical sites and the Data Coordinating Center, and all women provided written informed consent before participating.

Prior to randomization and surgery, participants underwent a standardized physical examination with assessment of POP according to the Pelvic Organ Prolapse Quantification (POP-Q) system [5]. POP severity was analyzed by POP-Q stage and by the leading edge of prolapse in centimeters relative to the hymen. In addition, participants completed assessments of generic healthrelated and condition-specific quality of life (QOL), administered by telephone by trained personnel from a central site. Pelvic symptoms and their functional impact were assessed by two validated measures: the Pelvic Floor Distress Inventory (PFDI) and the Pelvic Floor Impact Questionnaire (PFIQ) [4]. The PFDI is a 46item measure that yields three scale scores: Urinary Distress Inventory (UDI, 28 items, score 0-300), Pelvic Organ Prolapse Distress Inventory (POPDI, 16 items, score 0-300), and ColorectalAnal Distress Inventory (CRADI, 17 items, score 0-400). In addition, items from each scale can generate three subscale scores. The POPDI scale yields a general subscale ( 7 items, score 0-100), an anterior subscale (6 items, score $0-100$ ) and a posterior subscale ( 3 items, score 0-100). For each question in the PFDI, respondents are asked whether they usually experience a given symptom and, if so, how much it bothers them on a scale from 1 (not at all) to 4 (quite a bit). Higher scores indicate a higher level of symptoms, either more numerous symptoms or more bother related to symptoms.

The PFIQ focuses on specific activities of daily living and the extent to which they are affected by bladder, bowel, and/or pelvic symptoms. Three scales can be derived: the Incontinence Impact Questionnaire (IIQ), the Colorectal-Anal Impact Questionnaire (CRAIQ), and the Pelvic Organ Prolapse Impact Questionnaire (POPIQ), each with 31 items and scores from 0 to 400 . Respondents are asked how their bladder, bowel, and/or pelvic symptoms affect each of the activities on a scale from 1 (not at all) to 4 (quite a bit). Higher scores indicate greater (worse) functional impact.

The participant's age, race, body mass index (BMI) and prior incontinence or POP surgery (henceforth collectively referred to as (pelvic surgery') were thought a priori to be possible important predictors of current pelvic symptoms and QOL. Scores of the three primary subscales of the PFDI and PFIQ and the component (general, anterior and posterior) scores of the POPDI subscale were computed for each subject and stratified by prolapse stage. Mann-Whitney-Wilcoxon tests were used to compare scores between prolapse stages with stage II as the

Table 1 Clinical and demographic characteristics of 314 women by stage of POP

\begin{tabular}{|c|c|c|c|c|c|c|}
\hline & & \multicolumn{3}{|c|}{ POP stage } & \multirow[t]{2}{*}{ Total } & \multirow[t]{2}{*}{$p$-value } \\
\hline & & II & III & IV & & \\
\hline \multicolumn{2}{|l|}{$N$} & 43 & 211 & 60 & 314 & \\
\hline \multirow[t]{2}{*}{ Age } & Mean & 56 & 61 & 67 & 61 & $<0.001^{a}$ \\
\hline & $\begin{array}{l}\text { Std } \\
\text { dev }\end{array}$ & 12 & 10 & 8 & 10 & \\
\hline \multicolumn{7}{|l|}{ Race } \\
\hline \multicolumn{2}{|l|}{ Caucasian } & 39 & 195 & 53 & 287 & $0.110^{b, c}$ \\
\hline \multicolumn{2}{|c|}{ African American } & 0 & 10 & 6 & 16 & \\
\hline \multicolumn{2}{|l|}{ Other } & 5 & 4 & 2 & 11 & \\
\hline \multicolumn{7}{|l|}{ Ethnicity } \\
\hline \multicolumn{2}{|l|}{ Hispanic } & 0 & 8 & 1 & 9 & $0.520^{b}$ \\
\hline \multicolumn{2}{|c|}{ Non-Hispanic } & 43 & 203 & 59 & 305 & \\
\hline \multicolumn{7}{|c|}{$\begin{array}{l}\text { Prior surgery for } \\
\text { incontinence or POP }\end{array}$} \\
\hline Yes & & 25 & 79 & 22 & 126 & $0.040^{b}$ \\
\hline No & & 18 & 131 & 38 & 187 & \\
\hline \multirow{2}{*}{$\begin{array}{c}\text { Number of } \\
\text { cesarean } \\
\text { deliveries }\end{array}$} & Mean & 0.09 & 0.07 & 0.08 & 0.08 & $0.640^{d}$ \\
\hline & IQR & $0-0$ & $0-0$ & $0-0$ & $0-0$ & \\
\hline \multirow{2}{*}{$\begin{array}{l}\text { Number of } \\
\text { Vaginal } \\
\text { Deliveries }\end{array}$} & Mean & 2.88 & 2.92 & 3.34 & 2.99 & $0.100^{a}$ \\
\hline & IQR & $2-4$ & $2-3$ & $2-4$ & $2-4$ & \\
\hline \multicolumn{7}{|c|}{ Menopausal status } \\
\hline \multicolumn{2}{|c|}{ Pre-menopausal } & 10 & 23 & 3 & 36 & \\
\hline \multicolumn{2}{|c|}{ Post-menopausal } & 29 & 186 & 56 & 271 & \\
\hline \multicolumn{2}{|c|}{ Not sure/missing } & 4 & 2 & 1 & 7 & \\
\hline
\end{tabular}

POP: pelvic organ prolapse.

IQR: interquartile range.

${ }^{\text {a }}$ One-way analysis of variance.

${ }^{b}$ Fisher's Exact test.

c White/Caucasian vs. Black/African American.

${ }^{d}$ Kruskal-Wallis test. 
Table 2 PFDI and PFIQ subscale scores by POP stage

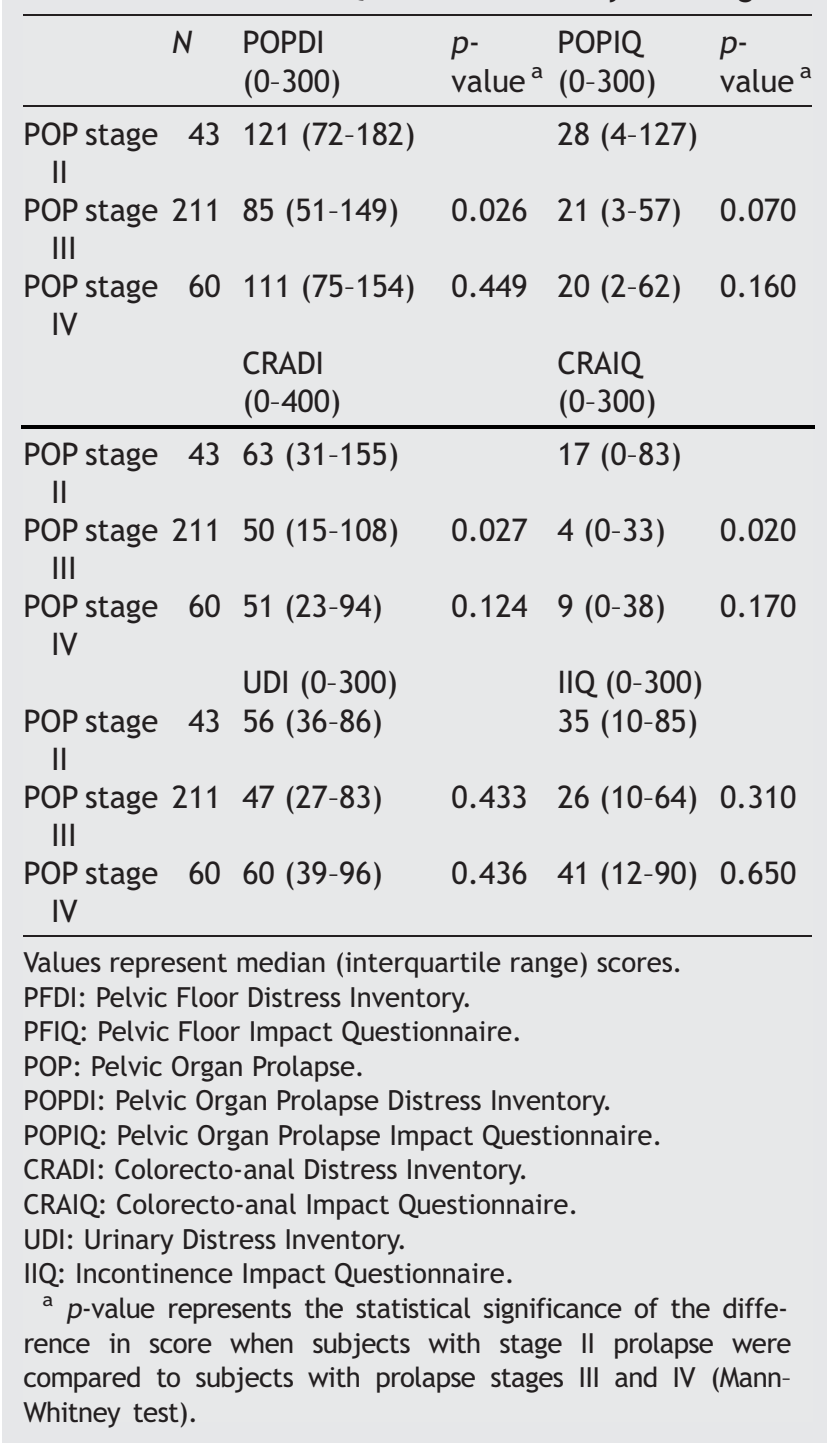

reference group, stratified by history of prior surgery. As women with stage II prolapse were significantly more likely to have had pelvic surgery (58.1\%) compared to women with stage III (37.6\%) and IV (36.7\%) prolapse, stages III and IV were combined in some analyses to examine the effect of prior pelvic surgery on symptom and life impact scores. Generalized linear regression models adjusting for age, race, and BMI were then fitted to examine the independent effects of collapsed POP stage variable on each subscale of the PFDI and PFIQ among women with and without prior pelvic surgery. An alpha level of 0.05 was applied for all hypothesis testing and all analyses were performed using SAS v9.1. Subjects without complete POP-Q or $\mathrm{PFDI} / \mathrm{PFIQ}$ data at baseline were excluded from these analyses.

\section{Results}

Baseline data from 314 CARE subjects were analyzed, representing $97.5 \%$ of all CARE subjects randomized. Table 1 details subjects' sociodemographic characteristics and past medical/surgical history. Notably, $40 \%$ of women had prior pelvic surgery, including 25 of 43 women (58.1\%) with stage II POP. Women with Stage II POP were significantly younger than women with more advanced stages of POP.

As detailed in Table 2, women with stage II prolapse had statistically significantly greater POPDI, CRADI and CRAIQ scores than women with stage III POP $(p<0.05)$; scores on the POPIQ followed the same pattern but were only marginally significant. Scores for women with stage IV POP were similar to Stage II scores. Women in all three POP-Q stages reported similar urinary symptoms (UDI) and urinary impact on daily activities (IIQ). Notably, while women with stage II and IV prolapse reported moderate levels of symptoms, the reported level of impact on daily activities remained quite low.

Table 3 details findings for the three prolapse subscales generated by the PFDI (general, anterior and posterior). As shown, a similar pattern of results was observed whereby women with stage II prolapse reported higher scores than women with more advanced prolapse.

Table 4 compares PFDI and PFIQ scores for women with stage II versus stages III or IV prolapse by prior pelvic surgery. Among women with prior surgery, women with stage II prolapse reported significantly more prolapse symptoms (POPDI) and marginally more impact (POPIQ) than women with more advanced prolapse. This contrast between the two groups is also statistically significant with respect to colorectal-anal symptoms (CRADI and CRAIQ) but not for urinary complaints (UDI and IIQ). There are no significant differences among women without prior pelvic surgery regardless of prolapse severity for any of the subscales. This was confirmed by regression models adjusting for age, BMI, and race that were constructed for PFDI and PFIQ scores by prior pelvic surgery. Among women with prior pelvic surgery, POPDI $(p=0.01)$, CRADI $(p<0.001)$, and CRAIQ $(p<0.003)$ all

Table 3 PFDI subscale POPDI scores by prolapse stage

\begin{tabular}{|c|c|c|c|c|c|c|}
\hline & $\begin{array}{l}\text { General subscale } \\
\text { (range } 0-100 \text { ) }\end{array}$ & $p$-value ${ }^{a}$ & $\begin{array}{l}\text { Anterior subscale } \\
\text { (range } 0-100 \text { ) }\end{array}$ & $p$-value ${ }^{a}$ & $\begin{array}{l}\text { Posterior subscale } \\
\text { (range } 0-100 \text { ) }\end{array}$ & $p$-value ${ }^{a}$ \\
\hline POP stage II $(N=43)$ & $57.1(21.4-78.6)$ & & $25.0(8.3-50.0)$ & & $25.0(8.3-91.7)$ & \\
\hline POP stage III $(N=210)$ & $42.9(25.0-64.3)$ & 0.177 & $20.8(4.2-54.2)$ & 0.639 & $16.7(0.0-33.3)$ & 0.005 \\
\hline POP stage IV $(N=60)$ & $50.0(32.1-71.4)$ & 0.639 & $33.3(10.4-58.3)$ & 0.302 & $25.0(0.0-37.5)$ & 0.030 \\
\hline
\end{tabular}

Table values represent median (interquartile range).

PFDI: Pelvic Floor Distress Inventory.

POPDI: Pelvic Organ Prolapse Distress Inventory.

POP: Pelvic Organ Prolapse.

${ }^{a} p$-values represent statistical significance of difference in subscale score when subjects with stage II prolapse were compared to subjects with prolapse stages III and IV. 
Table 4 Comparison of scores from subjects with stage II prolapse compared to those from subjects with stages III or IV, by prior surgery for incontinence or POP

\begin{tabular}{|c|c|c|c|}
\hline & POP stage & Score & $p$-value ${ }^{a}$ \\
\hline \multicolumn{4}{|l|}{ Prior surgery } \\
\hline \multirow[t]{2}{*}{ POPDI, range $0-300$} & II & $156(101-202)$ & 0.004 \\
\hline & III or IV & $95(54-158)$ & \\
\hline \multirow[t]{2}{*}{ POPIQ, range $0-400$} & II & $52(8-144)$ & 0.090 \\
\hline & III or IV & $25(6-54)$ & \\
\hline \multirow[t]{2}{*}{ CRADI, range 0-400 } & II & $117(58-188)$ & $<0.001$ \\
\hline & III or IV & $48(17-100)$ & \\
\hline \multirow[t]{2}{*}{ CRAIQ, range $0-400$} & II & $61(4-135)$ & 0.004 \\
\hline & III or IV & $5(0-33)$ & \\
\hline \multirow[t]{2}{*}{ UDI Range 0-300 } & II & $68(38-102)$ & $<0.300$ \\
\hline & III or IV & $56(33-90)$ & \\
\hline \multirow[t]{2}{*}{ IIQ, range $0-400$} & II & $43(11-114)$ & 0.200 \\
\hline & III or IV & $27(11-58)$ & \\
\hline \multicolumn{4}{|l|}{ No prior surgery } \\
\hline \multirow[t]{2}{*}{ POPDI, range 0-300 } & II & $85(44-121)$ & 0.440 \\
\hline & III or IV & $88(57-146)$ & \\
\hline \multirow{2}{*}{ POPIQ, range $0-400$} & II & $23(4-101)$ & 0.500 \\
\hline & III or IV & $19(0-67)$ & \\
\hline \multirow[t]{2}{*}{ CRADI, range $0-400$} & II & $30(17-53)$ & 0.200 \\
\hline & III or IV & $50(17-105)$ & \\
\hline \multirow[t]{2}{*}{ CRAIQ, range $0-400$} & II & $4(0-29)$ & 0.970 \\
\hline & III or IV & $4(0-34)$ & \\
\hline \multirow[t]{2}{*}{ UDI, range $0-400$} & II & $44(36-67)$ & 0.440 \\
\hline & III or IV & $48(29-84)$ & \\
\hline \multirow[t]{2}{*}{ IIQ, range $0-400$} & II & $35(10-54)$ & 0.690 \\
\hline & III or IV & $32(10-79)$ & \\
\hline
\end{tabular}

Values represent median (interquartile range).

PFDI: Pelvic Floor Distress Inventory.

PFIQ: Pelvic Floor Impact Questionnaire.

POPDI: Pelvic Organ Prolapse Distress Inventory.

POPIQ: Pelvic Organ Prolapse Impact Questionnaire.

CRADI: Colorecto-anal Distress Inventory.

CRAIQ: Colorecto-anal Impact Questionnaire.

UDI: Urinary Distress Inventory.

IIQ: Incontinence Impact Questionnaire.

${ }^{a} p$-value represents significance of the test for difference between these scores when subjects with stage II prolapse were compared to subjects with prolapse stages III or IV.

varied significantly with POP stage. In contrast, among women without prior pelvic surgery, symptoms and QOL did not vary with POP stage.

When prolapse was analyzed by the position of the leading edge of prolapse, rather than by POP stage, similar results were found (data not shown).

\section{Comment}

The most important finding of this study is that a history of pelvic surgery has a significant influence on the relationship between the stage of POP and the severity of pelvic symptoms and impact on quality of life.

Several observations can be made regarding women with stage II prolapse and prior pelvic surgery, who are now presenting for another surgical procedure for POP correction. It may be that those women have a lower threshold for discomfort and diminished quality of life before they are willing to undergo further surgery, i.e. women who previously had pelvic surgery may have different expectations and find symptoms more bothersome if they persist or recur. Alternatively, women with stage II POP may be more driven by the presence of pelvic symptoms to undergo reoperation, i.e., patients with stage II POP planning abdominal sacrocolpopexy may be only those with remarkable urinary and bowel symptoms concurrent with their relatively mild prolapse. Women with more advanced prolapse, with or without prior surgery, may be driven by the physical presence of prolapse more than by the bother and life impact of their other pelvic symptoms.

Finally, surgeon selection bias is likely to be present, since by definition these are patients with lower stages of POP who have been offered transabdominal prolapse repair. Specifically, it is likely that surgeons offer abdominal sacrocolpopexy to women with stage II POP who have significant symptoms and/or who have already had incontinence or POP repair. It may be that women with stage II POP and mild symptoms may be more likely to be offered transvaginal repair and would not be CARE participants. It will be especially interesting to observe these subjects with stage II POP to determine whether preoperative symptoms and bother resolve after surgery, or whether their symptoms represent something that prolapse repair does not address.

Although patient and surgeon selection biases probably explain much of our results, our findings do mirror those of several other studies conducted in other patient populations, that have demonstrated a relatively weak association between pelvic symptoms and the stage of prolapse. For example, Burrows et al. [6] found that patients with incontinence (stress and urge) had less advanced prolapse. Ellerkmann et al. [7] reported weak associations between voiding difficulty and increasing severity of anterior and apical prolapse, and between defecatory dysfunction, incomplete evacuation, digital manipulation for defecation and worsening posterior vaginal prolapse. Mouritsen [8] also reported similarly weak correlations between prolapse severity and all pelvic symptoms.

The weak association between PFDI/PFIQ scores and prolapse severity may also be related to the fact that POP$Q$ staging reflects only part of the clinical reality and cannot fully represent the function of the pelvic floor, urinary, or anorectal systems and the way in which symptoms impact individual patients. In addition, since the PFDI and PFIQ scales, during their initial validation in patients with stage III and IV prolapse, showed only moderate correlations with semi-quantitative measures of symptom severity such as bladder and bowel diaries, it is possible that our current tools cannot yet accurately reflect the most bothersome symptoms that patients experience in association with prolapse. Our results further emphasize the need for clinicians to assess the combination of clinical findings and symptom experience to fully understand how women are affected by pelvic floor dysfunction.

Strengths of this study include the large sample size, standardized evaluation, and data collection by experienced interviewers. Limitations include the lack of a control group and the heterogeneity of the study population. More homogeneous results might have been found in a study population limited to women undergoing prolapse surgery for the first time. However, study participants do represent a 
range of patients that clinicians encounter in their daily practices. In lieu of a control group, postoperative findings from this study population will be studied to learn more about which preoperative symptoms resolve and which persist, and may be useful in patient counseling.

\section{Acknowledgment}

Supported by grants from the National Institute of Child Health and Human Development (U01 HD41249, U10 HD41268, U10 HD41250, U10 HD41260, U10 Hd41263, U10 HD41269, U10 HD41267).

\section{References}

[1] Boyles SH, Weber AM, Meyn L. Procedures for pelvic organ prolapse in the United States, 1979-1997. Am J Obstet Gynecol 2003; 188:108-15.

[2] Brubaker L, Cundiff G, Fine P, Nygaard I, Richter H, Visco A. A randomized trial of colpopexy and urinary reduction efforts (CARE): design and methods. Control Clin Trials 2003; 24(5):629-42.
[3] Brubaker L, Cundiff G, Fine P, Nygaard I, Richter H, Visco A. Abdominal sacrocolpopexy with Burch colposuspension to reduce urinary stress incontinence. N Engl J Med 2006; 354:1557-66.

[4] Barber MD, Kuchibhatla MN, Pieper CF, Bump RC. Psychometric evaluation of 2 comprehensive condition-specific quality of life instruments for women with pelvic floor disorders. Am J Obstet Gynecol 2001; 185(6):1388-95.

[5] Bump RC, Mattiasson A, Bo K, Delancey JO, Klarskov P. The standardization of terminology of female pelvic organ prolapse and pelvic floor dysfunction. Am J Obstet Gynecol 1996;175 (1):10-7.

[6] Burrows LJ, Meyn LA, Walters MD, Weber AM. Pelvic symptoms in women with pelvic organ prolapse. Obstet Gynecol 2004;104 (1):982-8.

[7] Ellerkmann RM, Cundiff GW, Melick CF, Nihira MA, Leffler K, Bent $A E$. Correlation of symptoms with location and severity of pelvic organ prolapse. Am J Obstet Gynecol 2001; 185(6):1332-7 [discussion 1337-8].

[8] Mouritsen L, Larsen JP. referred with pelvic organ prolapse. Int Urogynecol J 2003; 14(2):122-7. 